\title{
Phase-Tuned Entangled State Generation between Distant Spin Qubits
}

\author{
R. Stockill, ${ }^{1}$ M. J. Stanley, ${ }^{1}$ L. Huthmacher, ${ }^{1}$ E. Clarke, ${ }^{2}$ M. Hugues, ${ }^{3}$ A. J. Miller, ${ }^{4}$ \\ C. Matthiesen, ${ }^{1, *}$ C. Le Gall, ${ }^{1}$ and M. Atatüre ${ }^{1, \dagger}$ \\ ${ }^{1}$ Cavendish Laboratory, University of Cambridge, JJ Thomson Avenue, Cambridge CB3 OHE, United Kingdom \\ ${ }^{2}$ EPSRC National Centre for III-V Technologies, University of Sheffield, Sheffield S1 3JD, United Kingdom \\ ${ }^{3}$ Université Côte d'Azur, CNRS, CRHEA, rue Bernard Grégory, Valbonne 06560, France \\ ${ }^{4}$ Quantum Opus, LLC, 45211 Helm St., Plymouth, Michigan 48170, USA
}

(Received 11 February 2017; published 7 July 2017)

\begin{abstract}
Quantum entanglement between distant qubits is an important feature of quantum networks. Distribution of entanglement over long distances can be enabled through coherently interfacing qubit pairs via photonic channels. Here, we report the realization of optically generated quantum entanglement between electron spin qubits confined in two distant semiconductor quantum dots. The protocol relies on spin-photon entanglement in the trionic $\Lambda$ system and quantum erasure of the Raman-photon path information. The measurement of a single Raman photon is used to project the spin qubits into a joint quantum state with an interferometrically stabilized and tunable relative phase. We report an average Bell-state fidelity for $\left|\psi^{(+)}\right\rangle$and $\left|\psi^{(-)}\right\rangle$states of $61.6 \pm 2.3 \%$ and a record-high entanglement generation rate of $7.3 \mathrm{kHz}$ between distant qubits.
\end{abstract}

DOI: 10.1103/PhysRevLett.119.010503

The refutation of local realism in favor of a nonlocal quantum theory $[1,2]$ has been supported by a number of decisive experiments using entangled pairs of photons [3,4], atoms $[5,6]$, and solid-state systems $[7,8]$. While photonic links are essential to close loopholes in Bell tests by removing the requirement of spatial proximity for entanglement creation, they also permit flexible arrangements in which distant systems with a spin-photon interface can be entangled. The emergence of entanglement as a central resource in quantum sensing, communication, and computation [9] benefits from this flexibility, where matter qubits coherently coupled to well-defined optical modes provide the elementary constituents of a distributed quantum network [10]. Accordingly, the creation of entangled states between distant qubits has received intense experimental attention in a number of physical systems [6,11-16]. The operation rate of any quantum network would ultimately depend upon the strength of the light-matter coupling between stationary and flying qubits. In this regard, indium-gallium-arsenide (InGaAs) quantum dots (QDs) feature a particularly high oscillator strength of the spin-photon interface, with the potential for high rates of entanglement distribution [17]. Matter qubits can be realized using confined electrons [18], heavy holes [19], or dark excitons [20] in these systems. While quantum correlations have been observed between distant heavy hole spins [15], it is the electron that offers the longest coherence time in this system to date [21-23].

In this Letter, we present optical generation of nonlocal quantum-entangled states between two distant nodes formed by electron spins confined in separate QDs. Through a single-photon state projection protocol [24] and the bright narrow-linewidth emission available from QDs [25], we realize an entanglement generation rate of $7.3 \mathrm{kHz}$, the highest rate to date. Further, with full control over the singlephoton interference, we create remote entangled states with an arbitrary phase. Prior to this point, phase control of the generated entangled state had only been demonstrated for atomic nodes located in the same trap [13]. Together with local gate operation times of a few picoseconds [18], microsecond-long spin coherence [21-23], and the current state-of-the-art for on-chip integration [17,26], this Letter represents progress towards small-scale on-chip quantum networks with high bandwidth operation.

The entanglement scheme we employ was proposed by Cabrillo et al. [24], which relies on the weak phasecoherent excitation of two $\Lambda$ systems and the subsequent detection of a single state-projecting Raman photon $[13,15]$. First, the $\Lambda$ systems (with spin- $1 / 2$ ground states $\left|\uparrow_{i}\right\rangle$ and $\left.\left|\downarrow_{i}\right\rangle\right)$ are excited from the state $\left|\downarrow_{i}\right\rangle$, resulting in a Raman scattering process, with total amplitude $\sqrt{p} \ll 1$ set by the excitation pulse area and the excited state branching ratio. This process entangles the ground state of each system with the occupation of the Raman-photon mode [27-29]. Overlapping the Raman modes on a 50:50 beam splitter erases the which-path information, such that the total entangled spin-photon state is given by

$$
\begin{aligned}
|\Psi\rangle= & (1-p)\left|\downarrow_{A} \downarrow_{B}\right\rangle\left|0_{1} 0_{2}\right\rangle \\
& +\sqrt{p(1-p) / 2}\left(e^{i \phi_{A}}\left|\uparrow_{A} \downarrow_{B}\right\rangle+e^{i \phi_{B}}\left|\downarrow_{A} \uparrow_{B}\right\rangle\right)\left|1_{1} 0_{2}\right\rangle \\
& +\sqrt{p(1-p) / 2}\left(e^{i \phi_{A}}\left|\uparrow_{A} \downarrow_{B}\right\rangle-e^{i \phi_{B}}\left|\downarrow_{A} \uparrow_{B}\right\rangle\right)\left|0_{1} 1_{2}\right\rangle \\
& +p / \sqrt{2} e^{i\left(\phi_{A}+\phi_{B}\right)}\left|\uparrow_{A} \uparrow_{B}\right\rangle\left(\left|2_{1} 0_{2}\right\rangle-\left|0_{1} 2_{2}\right\rangle\right) .
\end{aligned}
$$

Here, the indices in the photonic number states designate the beam splitter output mode ( 1 or 2$). \phi_{A}\left(\phi_{B}\right)$ is the 
optical phase accumulated along the path going through system $A$ (system $B$ ). The state is sorted according to zero-, one-, and two-photon components.

Detection of a one-photon contribution projects the qubits into the maximally entangled state $\left.\left(\left|\uparrow_{A} \downarrow_{B} \pm e^{i \Delta \phi}\right| \downarrow_{A} \uparrow_{B}\right\rangle\right) / \sqrt{2}$, with the sign depending on the output port that registers the photon and $\Delta \phi=\phi_{B}-\phi_{A}$. For $\Delta \phi=0$, these states correspond to the Bell states $\left|\psi^{( \pm)}\right\rangle$. With a probability of $p^{2}$, both qubits undergo a spin-flip process, resulting in a two-photon state after the beam splitter. Without number-resolving detectors, and in the presence of optical losses, we cannot distinguish this component from the single-photon states, setting an intrinsic error in the Bell state generation of $p$. Optimal operation requires a compromise between this error and the probabilistic entangled-state generation rate $2 p(1-p)$.

The experimental realization of this protocol is introduced in Fig. 1. Single electrons confined in two InGaAs $\mathrm{QDs}, \mathrm{QD}_{A}$, and $\mathrm{QD}_{B}$, located approximately two meters apart in separate cryostats, provide the stationary nodes. The 4-T magnetic field, applied perpendicular to the QD growth axis, lifts the spin degeneracy of the ground and

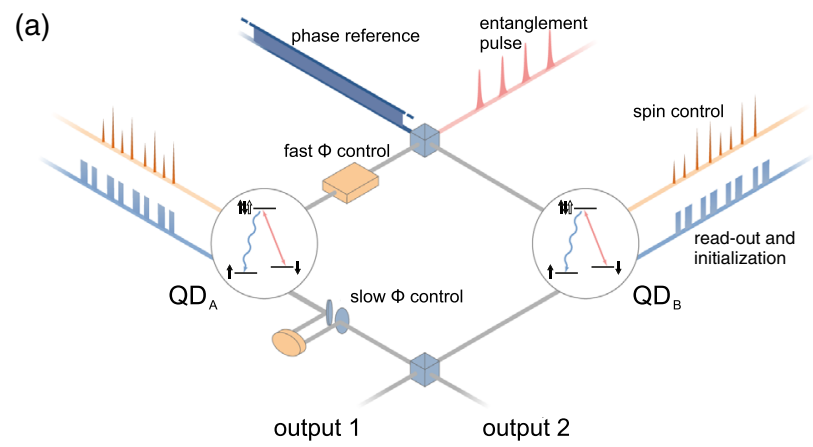

(b)

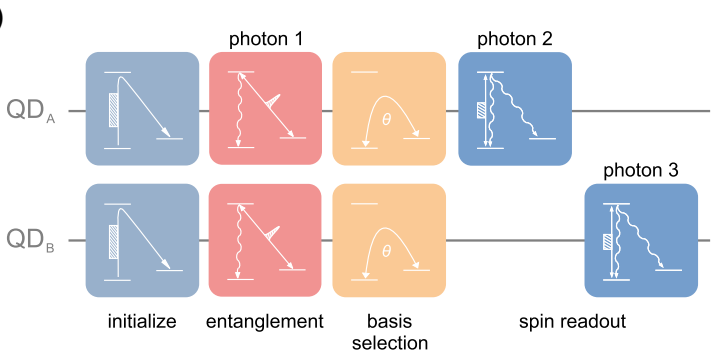

FIG. 1. Experimental setup and sequence for detecting entanglement between distant spin qubits. (a) Sketch of the optical interferometer with the QD nodes. Optical pulses, labeled entanglement, read-out and initialization, and spin control are used, together with a continuous-wave phase reference laser. Active phase stabilization utilizes a retroreflector on a piezo stack and a phase electro-optic modulator. (b) Entanglement verification sequence. The spins are initialized in the $\left|\downarrow_{A} \downarrow_{B}\right\rangle$ state. The entanglement creation pulse is followed by coherent rotations of both qubits when measuring in an arbitrary basis. Further $\pi$ rotations of the qubits are added to cycle through the four twospin combinations in a given basis. Resonant excitation of the blue transition is used for spin read-out. excited states within each node. The 25-GHz-split electron spin ground states, together with one of the two excited states, form a $\Lambda$ system [Fig. 1(a)]. The two optical transitions, at $968 \mathrm{~nm}$ in our case, are distinguished by their frequency; the lower-energy and higher-energy transitions are denoted as red and blue, respectively. The entangled state phase $\Delta \phi$ is defined by the general architecture of our setup: a Mach-Zehnder interferometer (MZI), where the mirrors in the two arms are replaced by the two QDs [Fig. 1(a)].

The measurement sequence is illustrated in Fig. 1(b). Both spins are optically prepared in the spin-down state with $\approx 97 \%$ fidelity. We apply a 160 -ps-long pulse to the red transition of each QD to generate the state-projecting Raman photon. The state-change probability $p$ is set to $7 \%$ in order to suppress the error in the entangled state due to simultaneous spin-flip events. Having detected a Raman photon [photon 1 in Fig. 1(b)], the state is reconstructed from spin correlations in different measurement bases [photons 2 and 3 in Fig. 1(b)]. We combine local spinrotation with the read-out of the spin-up state to reconstruct the population of $\left\{\left|\downarrow_{A} \downarrow_{B}\right\rangle,\left|\downarrow_{A} \uparrow_{B}\right\rangle,\left|\uparrow_{A} \downarrow_{B}\right\rangle,\left|\uparrow_{A} \uparrow_{B}\right\rangle\right\}$ in four iterations of the sequence. The read-out of each QD is performed with 8-ns-long pulses resonant with the blue transition. An optional coherent rotation of both electron spins allows correlations to be measured in a rotated basis. These rotations occur within the inhomogeneous dephasing time $\left(T_{2}^{*}\right)$ of $\sim 1.2 \mathrm{~ns}$ after the entanglement pulse, during which, the phase of the two-spin state is preserved $[23,30]$.

Spin initialization, entanglement, and read-out pulses are derived from frequency-stabilized single-mode lasers, using fiber-based electro-optic intensity modulators. Local spin rotations are performed optically [18], using $1-\mathrm{THz}$ red-detuned pulses, picked from a mode-locked Ti:sapphire laser with acousto-optic modulators. The experiment has a sequence length of 78.9 ns and a repetition rate of 10.9 MHz [31], clocked by the Ti:sapphire laser. We use a far-off resonance laser to monitor the phase of the MZI continuously. Electro-optic phase modulation and piezobased compensation [fast and slow $\phi$ control in Fig. 1(a)] allow us to actively stabilize to $\approx 3$ degree over a dc $-1.5 \mathrm{kHz}$ range. The working point of the MZI and the timing of the spin rotations are arranged such that we can determine a priori the phase of the entangled state by monitoring the interference of coherent Rayleigh scattering from the two emitters [31]. Gratings and Fabry-Pérot filters are used to separate the phase-reference laser and the red and blue QD fluorescence onto six single-photon detectors. A time-to-digital converter time tags photon detection events for analysis [31].

Projecting a well-defined entangled state with a stationary relative phase requires identical $\Lambda$ systems for the two QDs [32]. First, we evaluate the ground state energy splitting using Ramsey interferometry [Fig. 2(a)] and adjust the Zeeman energy of the spin qubits by fine-tuning the 

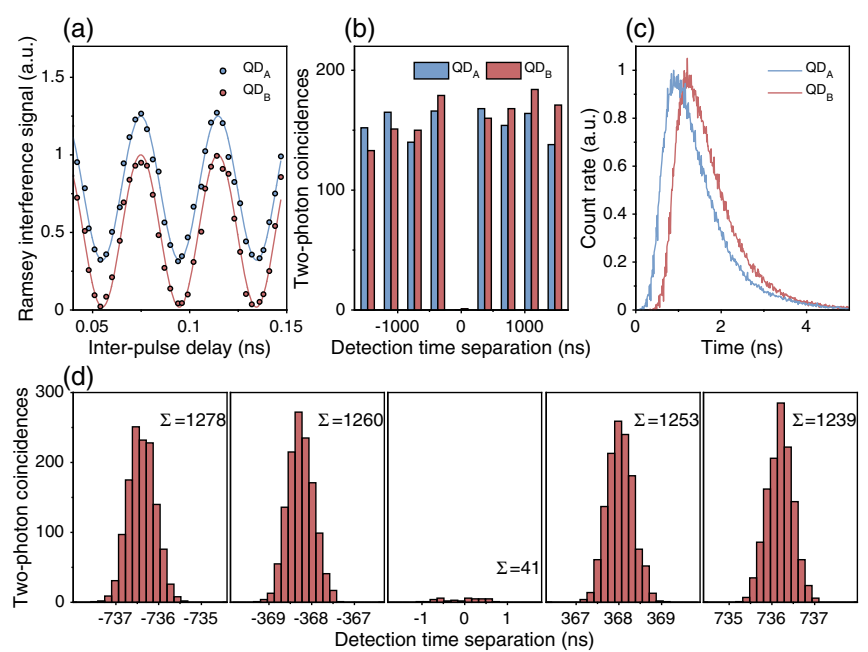

FIG. 2. Overlap of the optical and spin properties. (a) Ramsey interference fringes for both QDs (the data and curves have been offset for clarity). Sinusoidal fits to the data show electron spin splittings of $2 \pi \times 25.2 \pm 0.1 \mathrm{GHz}$ for $\mathrm{QD}_{A}$ and $2 \pi \times 25.1 \pm 0.1 \mathrm{GHz}$ for $\mathrm{QD}_{B}$. (b) Two-photon correlation measurements of the Raman emission generated by the entanglement pulse for each QD, with $0.78 \%$ average background. (c) Radiative decay measurements of the Raman transitions (offset for clarity). (d) Raman two-photon indistinguishability measurement with the same count rate from each QD. The five peaks closest to zero-time delay are displayed; the indistinguishability is $93 \pm 1 \%$.

external magnetic fields around $4 \mathrm{~T}$. The spin precession frequencies are closely matched at around $2 \pi \times 25.1 \mathrm{GHz}$, ensuring that the phase $\Delta \phi$ is stationary in the laboratory frame. Under these conditions, the Zeeman energy far exceeds the optical linewidth, and each QD acts as a photon turnstile, whereby a Raman scattering process shelves the electron spin, preventing subsequent excitation. Figure 2(b) displays the second-order autocorrelation measurements of the Raman scattering from each QD, following the entanglement pulse. The antibunching is limited in our case by laser background, which contributes 1 in 150 events on average, consistent with the single twophoton event recorded at zero time delay. In parallel, we must consider the optical mode matching between the two QDs. Figure 2(c) shows very similar excited state lifetimes: $727 \pm 10 \mathrm{ps}(742 \pm 10 \mathrm{ps})$ for $\mathrm{QD}_{A}\left(\mathrm{QD}_{B}\right)$, guaranteeing photon-wave-packet overlap in time, while a static electric field applied across each sample is used to overlap the photons spectrally via the Stark effect. Our setup filters the phonon-assisted emission $(\approx 10 \%)$ that occurs outside of a well-defined frequency mode [33,34]. We implement a Hong-Ou-Mandel experiment $[35,36]$ to quantify the quality of the quantum erasure process by cross correlating the Raman photons in the two beam splitter outputs following the entanglement pulse. The central five peaks of the correlation are shown in Fig. 2(d). The small number of coincidences in the central peak is the signature of photon coalescence, owing to the indistinguishability of the input

modes, revealing a two-photon interference visibility of $93 \pm 1 \%$. Imperfect optical elements account for a $4 \%$ reduction of the visibility in our setup. We only consider photons within a 1.2-ns window from the start of the entanglement pulse, limiting the effect of inhomogeneous spin dephasing on Raman photon distinguishability [37], with the outcome that we reject $42 \%$ of the emission events.

In Fig. 3, we reconstruct the projected two-spin state from the correlation of three-photon coincidence events. We first measure the state in the population basis, parallel to the external magnetic field [Fig. 3(a)]. We find that the detection of a Raman photon predicts an antisymmetric spin population with a probability of $85.7 \pm 3.8 \%$. The uncertainty is set by the shot noise of the 603 three-photon events that contribute to the data in Fig. 3(a). The presence of population in the $\left|\uparrow_{A} \uparrow_{B}\right\rangle$ state is intrinsic to the

(a)

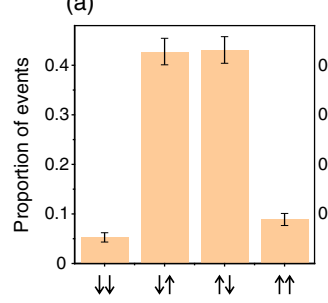

(b)
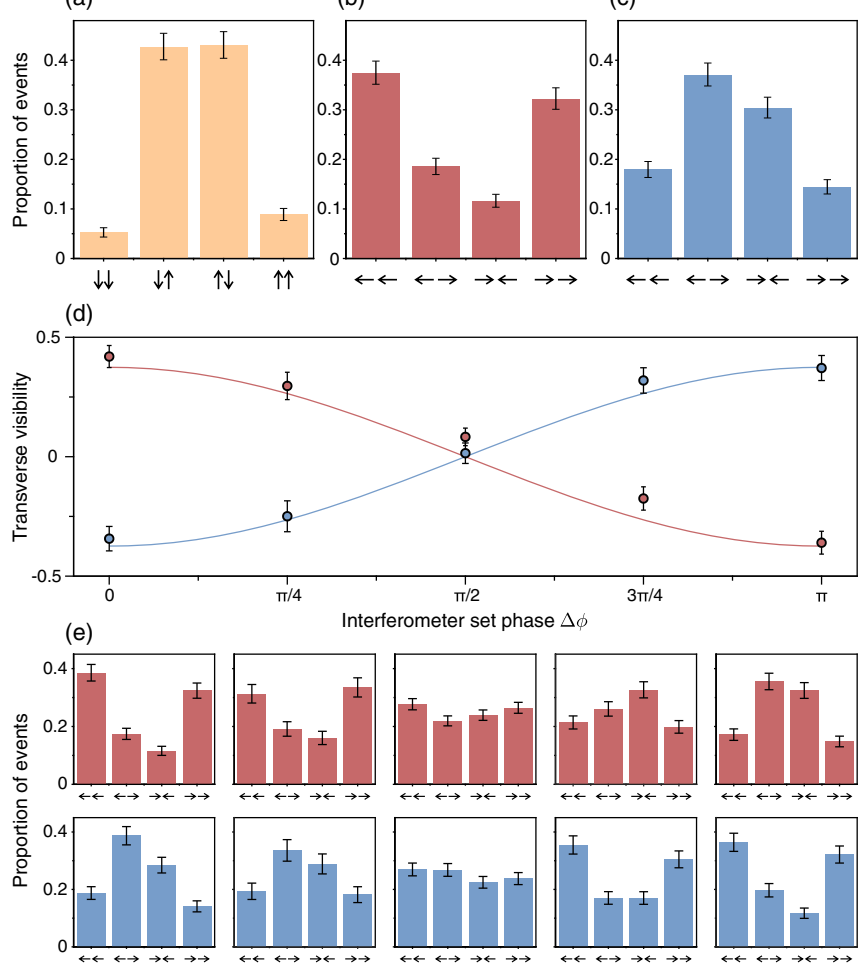

FIG. 3. Joint spin-state reconstruction through three-photon coincidence events. (a) Joint spin-state population conditional on a single Raman photon detection event. An antisymmetric population is retrieved with a probability of $85.7 \pm 3.8 \%$. The error bars represent the statistical uncertainty drawn from the 603 three-photon events that were used to reconstruct the population. (b),(c) Spin correlations in the transverse basis for projection of $\left|\psi^{(+)}\right\rangle$(b) or $\left|\psi^{(-)}\right\rangle$(c). (d) Visibility in the transverse basis for varying optical phase between the interfering paths. A positive visibility corresponds to a correlated phase and negative to an anticorrelated phase. The curves are sinusoidal fits to the data. (e) Normalized coincidence events for a Raman photon detected on beam splitter output 1 (red histograms) or output 2 (blue histograms) for five different set points of the interferometer phase. 
entanglement generation scheme and follows the spin-flip probability $p$. Events in the $\left|\downarrow_{A} \downarrow_{B}\right\rangle$ state mainly result from imperfect spin rotation prior to the population read-out [31], as well as read-out laser leakage. Additional rotations in our pulse sequence [Fig. 1(b)] transfer the phase of the entangled state into correlated spin populations. We stabilize the Raman mode at either $\Delta \phi=0$ or $\Delta \phi=\pi$ to obtain maximum contrast in our state tomography. Figures 3(b) and 3(c) show correlation measurements in this transverse basis. For $\Delta \phi=0$, Raman-photon detection in output mode 1 generates the $\left|\psi^{(+)}\right\rangle$state, while detection in mode 2 generates $\left|\psi^{(-)}\right\rangle$. When the Raman mode is stabilized at $\Delta \phi=\pi$, output mode 1(2) heralds the creation of $\left|\psi^{(-)}\right\rangle\left(\left|\psi^{(+)}\right\rangle\right)$. In Figs. 3(b) and 3(c), the coincidence events are displayed accordingly, with panel $\mathrm{b}$ corresponding to projection of $\left|\psi^{(+)}\right\rangle$and panel c to $\left|\psi^{(-)}\right\rangle$.

In the rotated basis, the $\pi$ phase shift between the states generated at opposite output ports is apparent from the correlation histograms, revealing visibilities of $39.5 \pm 3.8 \%$ for $\left|\psi^{(+)}\right\rangle$[Fig. 3(b)] and 35.1 $\pm 3.8 \%$ for $\left|\psi^{(-)}\right\rangle$[Fig. 3(c)]. Owing to the rapid phase evolution of all but a subset of spin coherences in the density matrix [31], we can directly estimate the Bell-state fidelity from combining these visibilities with our population measurement. Overall, we find that a photon detection heralds either the $\left|\psi^{(+)}\right\rangle$ or $\left|\psi^{(-)}\right\rangle$state, with an average of $61.6 \pm 2.3 \%$ fidelity. For these results, the nonclassicality of the state is confirmed to 5 standard deviations of the mean. The measured fidelity can be understood by taking into account the contributions of the double spin-flip rate (limiting the fidelity to 93\%), the imperfect mode overlap (a further reduction of 4\%), the spin-state dephasing (a visibility reduction of 13\%), and the imperfect spin preparation and read-out (3\% and 6\%, respectively) [31]. The combination of these factors predict a fidelity of $76 \%$. The remaining source for our observed reduction below this value is electrical noise in each QD sample $[38,39]$, which alters the relative phase of the two optical excitations, and in turn, the phase of the entangled state [31].

In Figs. 3(d) and 3(e), we demonstrate direct control over the generated two-electron spin state. Figure 3(d) shows the extracted visibilities from transverse basis measurements for five different values of the entangled-state phase. The visibilities are drawn from the relative coincidence rates displayed in Fig. 3(e). We observe a sinusoidal variation in the visibility of the phase-basis correlations as the MZI phase $\Delta \phi$ is tuned from 0 to $\pi$. The results are partitioned according to the interferometer output that registers the Raman photon. For $\Delta \phi=0$, the first (second) output projects the spins to $\left|\psi^{(+)}\right\rangle\left(\left|\psi^{(-)}\right\rangle\right)$, resulting in a correlated (anticorrelated) population in the transverse measurement. As we move to $\Delta \phi=\pi / 2$, we project to $\left|\psi^{(+)} \pm \psi^{(-)}\right\rangle$, which does not exhibit transverse spin-spin correlations. At $\Delta \phi=\pi$, the relationships between the detection modes and the spin states are swapped, and the visibility along our measurement axis returns. This state control demonstrates the working principle of our experiment: quantum erasure imprints the interferometric phase between the Raman modes onto the nonlocal state shared between the two spins.

In Fig. 4, we compare our results with entanglement rates and state fidelities reported thus far for distant qubits using projection-by-measurement protocols. Figure 4(a) shows the Bell-state fidelities vs the entanglement generation rate for atomic qubits [11-13,40,41], nitrogen-vacancy centers $[8,14,42]$, superconducting qubits [16], and QD hole spins [15], along with our results. At $7.3 \mathrm{kHz}$, we are reporting the fastest distribution of entanglement between distant qubits in any system. Not requiring intermittent cooling or reloading steps, our protocol operates continually at a $10.9-\mathrm{MHz}$ attempt rate. Figure 4(b) compares the success probability, which factors out the intrinsic time scales of each physical system and the specific requirements of each control sequence. The single-photon protocol we use allows for a success probability of $6.7 \times 10^{-4}$, the highest reported for optically linked qubits. In particular, our demonstration benefits from superconducting nanowire single-photon detectors with a detection efficiency of $\sim 80 \%$ at $968 \mathrm{~nm}$, contributing to an overall average photon detection efficiency of $\sim 1 \%$. The success probability of the single-photon scheme is intimately linked to the Bell-state fidelity. The dashed red curves in Fig. 4 project our state fidelity over different entanglement generation rates (a) and generation probability (b), establishing the reach of our experimental protocol. Reducing double spin-flip events would increase the fidelity at the expense of lower scattering probabilities,

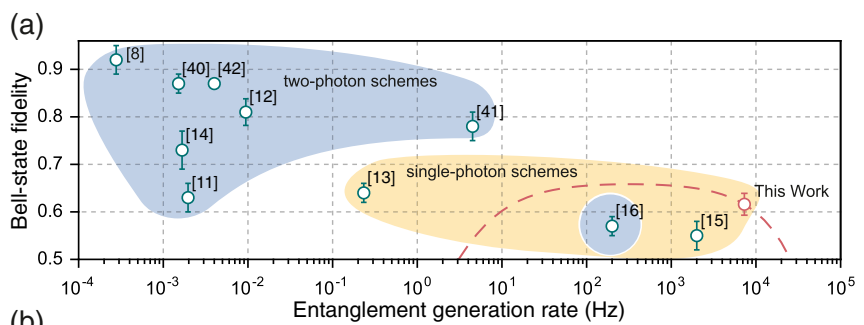

(b)

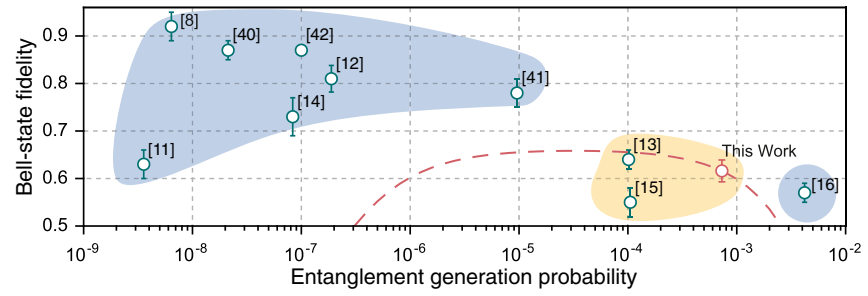

FIG. 4. Comparison of the measured fidelity and entanglementgeneration rate (a) or generation probability (b) between the electron spins in this Letter and other physical systems. The indices denote the reference of the work from which the figures were extracted. The shaded regions mark the distribution protocol used, either single- or two-photon detection state projection (yellow and blue, respectively). We note our quoted fidelity is not corrected for our imperfect state retrieval. 
accounting for the intrinsic and technical limitations discussed earlier, until we reach the $\approx 1-\mathrm{Hz}$ detector dark count rate where false heralds dominate.

In this Letter, we demonstrate optically generated quantum entanglement between two confined electron spins. In order to use this probabilistic scheme in computational protocols, a herald is necessary to allow a repeat-until-success approach. Owing to the short ensemble dephasing time of the electron spins, we apply the spin read-out sequence before the Raman photon reaches the detector. Local decoupling operations would preserve the coherence of the joint quantum state beyond the required propagation time of the state-heralding photon $(\approx 100 \mathrm{~ns}$ in our system) [21-23]. In order to extend to multiple nodes and realize fault-tolerant operation, we require both distribution rates within the storage time of the entangled state and additional memory qubits. Using current state-ofthe-art light-collection strategies [43,44], the entanglement rate could be improved to $130 \mathrm{kHz}$. This rate approaches the inverse of electron-spin coherence times in self-assembled QDs [21,23,45], a crucial benchmark for fault tolerant scalability [46]. With further improvement of this rate, the second spin of a QD molecule [47] could be utilized as a memory qubit. Alternatively, hybrid structures constituting systems with long coherence times, such as electrostatically defined QDs [48], could surpass this threshold.

We gratefully acknowledge financial support by the European Research Council ERC Consolidator Grant Agreement No. 617985 and the EPSRC National Quantum Technologies Programme No. NQIT EP/M013243/1. C. M. acknowledges Clare College, Cambridge, for financial support through a Junior Research Fellowship. We thank A. Imamoglu, B. Munro, and M. Weber for fruitful discussions and S. Topliss for technical assistance.

R. S., M. J. S., and L. H. contributed equally to this work.

*Present address: Department of Physics, University of California, Berkeley, California 94720, USA. †ma424@cam.ac.uk

[1] A. Einstein, B. Podolsky, and N. Rosen, Phys. Rev. 47, 777 (1935).

[2] J. S. Bell, Physics 1, 195 (1964).

[3] S. J. Freedman and J. F. Clauser, Phys. Rev. Lett. 28, 938 (1972).

[4] A. Aspect, J. Dalibard, and G. Roger, Phys. Rev. Lett. 49, 1804 (1982).

[5] M. A. Rowe, D. Kielpinski, V. Meyer, C. A. Sackett, W. M. Itano, C. Monroe, and D. J. Wineland, Nature (London) 409, 791 (2001).

[6] D. N. Matsukevich, P. Maunz, D. L. Moehring, S. Olmschenk, and C. Monroe, Phys. Rev. Lett. 100, 150404 (2008).

[7] M. Ansmann, H. Wang, R. C. Bialczak, M. Hofheinz, E. Lucero, M. Neeley, A. D. O'Connell, D. Sank, M. Weides, J. Wenner, A. N. Cleland, and J. M. Martinis, Nature (London) 461, 504 (2009).
[8] B. Hensen, H. Bernien, A. E. Dréau, A. Reiserer, N. Kalb, M. S. Blok, J. Ruitenberg, R. F. L. Vermeulen, R. N. Schouten, C. Abellán, W. Amaya, V. Pruneri, M. W. Mitchell, M. Markham, D. J. Twitchen, D. Elkouss, S. Wehner, T. H. Taminiau, and R. Hanson, Nature (London) 526, 682 (2015).

[9] W. K. Wootters, Phil. Trans. R. Soc. A 356, 1717 (1998).

[10] H. J. Kimble, Nature (London) 453, 1023 (2008).

[11] D. L. Moehring, P. Maunz, S. Olmschenk, K. C. Younge, D. N. Matsukevich, L.-M. Duan, and C. Monroe, Nature (London) 449, 68 (2007).

[12] J. Hofmann, M. Krug, N. Ortegel, L. Gerard, M. Weber, W. Rosenfeld, and H. Weinfurter, Science 337, 72 (2012).

[13] L. Slodička, G. Hétet, N. Röck, P. Schindler, M. Hennrich, and R. Blatt, Phys. Rev. Lett. 110, 083603 (2013).

[14] H. Bernien, B. Hensen, W. Pfaff, G. Koolstra, M. S. Blok, L. Robledo, T. H. Taminiau, M. Markham, D. J. Twitchen, L. Childress, and R. Hanson, Nature (London) 497, 86 (2013).

[15] A. Delteil, Z. Sun, W.-b. Gao, E. Togan, S. Faelt, and A. Imamoglu, Nat. Phys. 12, 218 (2015).

[16] A. Narla, S. Shankar, M. Hatridge, Z. Leghtas, K. M. Sliwa, E. Zalys-Geller, S. O. Mundhada, W. Pfaff, L. Frunzio, R. J. Schoelkopf, and M. H. Devoret, Phys. Rev. X 6, 031036 (2016).

[17] P. Lodahl, S. Mahmoodian, and S. Stobbe, Rev. Mod. Phys. 87, 347 (2015).

[18] D. Press, T. D. Ladd, B. Zhang, and Y. Yamamoto, Nature (London) 456, 218 (2008).

[19] D. Brunner, B. D. Gerardot, P. A. Dalgarno, G. Wüst, K. Karrai, N. G. Stoltz, P. M. Petroff, and R. J. Warburton, Science 325, 70 (2009).

[20] I. Schwartz, E. R. Schmidgall, L. Gantz, D. Cogan, E. Bordo, Y. Don, M. Zielinski, and D. Gershoni, Phys. Rev. X 5, 011009 (2015).

[21] D. Press, K. De Greve, P. L. McMahon, T. D. Ladd, B. Friess, C. Schneider, M. Kamp, S. Höfling, A. Forchel, and Y. Yamamoto, Nat. Photonics 4, 367 (2010).

[22] A. Bechtold, D. Rauch, F. Li, T. Simmet, P.-L. Ardelt, A. Regler, K. Müller, N. A. Sinitsyn, and J. J. Finley, Nat. Phys. 11, 1005 (2015).

[23] R. Stockill, C. Le Gall, C. Matthiesen, L. Huthmacher, E. Clarke, M. Hugues, and M. Atatüre, Nat. Commun. 7, 12745 (2016).

[24] C. Cabrillo, J. I. Cirac, P. Garcia-Fernandez, and P. Zoller, Phys. Rev. A 59, 1025 (1999).

[25] A. V. Kuhlmann, J. H. Prechtel, J. Houel, A. Ludwig, D. Reuter, A. D. Wieck, and R. J. Warburton, Nat. Commun. 6, 8204 (2015).

[26] I. J. Luxmoore, N. A. Wasley, A. J. Ramsay, A. C. T. Thijssen, R. Oulton, M. Hugues, S. Kasture, V. G. Achanta, A. M. Fox, and M.S. Skolnick, Phys. Rev. Lett. 110, 037402 (2013).

[27] W. B. Gao, P. Fallahi, E. Togan, J. Miguel-Sanchez, and A. Imamoglu, Nature (London) 491, 426 (2012).

[28] K. De Greve, L. Yu, P. L. McMahon, J. S. Pelc, C. M. Natarajan, N. Y. Kim, E. Abe, S. Maier, C. Schneider, M. Kamp, S. Hoefling, R. H. Hadfield, A. Forchel, M. M. Fejer, and Y. Yamamoto, Nature (London) 491, 421 (2012).

[29] J. R. Schaibley, A. P. Burgers, G. A. McCracken, L. M. Duan, P. R. Berman, D. G. Steel, A. S. Bracker, D. Gammon, and L. J. Sham, Phys. Rev. Lett. 110, 167401 (2013). 
[30] I. A. Merkulov, A. L. Efros, and M. Rosen, Phys. Rev. B 65, 205309 (2002).

[31] See Supplemental Material at http://link.aps.org/ supplemental/10.1103/PhysRevLett.119.010503 for additional data, description, and analysis of results in this Letter.

[32] G. Vittorini, D. Hucul, I. V. Inlek, C. Crocker, and C. Monroe, Phys. Rev. A 90, 040302 (2014).

[33] C. Matthiesen, M. Geller, C. H. H. Schulte, C. Le Gall, J. Hansom, Z. Li, M. Hugues, E. Clarke, and M. Atatüre, Nat. Commun. 4, 1600 (2013).

[34] J. Hansom, C. H. H. Schulte, C. Matthiesen, M. J. Stanley, and M. Atatüre, Appl. Phys. Lett. 105, 172107 (2014).

[35] C. K. Hong, Z. Y. Ou, and L. Mandel, Phys. Rev. Lett. 59, 2044 (1987).

[36] E. B. Flagg, A. Muller, S. V. Polyakov, A. Ling, A. Migdall, and G. S. Solomon, Phys. Rev. Lett. 104, 137401 (2010).

[37] C. Santori, D. Fattal, K.-M. M. C. Fu, P. E. Barclay, and R. G. Beausoleil, New J. Phys. 11, 123009 (2009).

[38] A. V. Kuhlmann, J. Houel, A. Ludwig, L. Greuter, D. Reuter, A. D. Wieck, M. Poggio, and R. J. Warburton, Nat. Phys. 9, 570 (2013).

[39] C. Matthiesen, M. J. Stanley, M. Hugues, E. Clarke, and M. Atatüre, Sci. Rep. 4, 4911 (2014).

[40] P. Maunz, S. Olmschenk, D. Hayes, D. N. Matsukevich, L.-M. Duan, and C. Monroe, Phys. Rev. Lett. 102, 250502 (2009).
[41] D. Hucul, I. V. Inlek, G. Vittorini, C. Crocker, S. Debnath, S. M. Clark, and C. Monroe, Nat. Phys. 11, 37 (2014).

[42] W. Pfaff, B. J. Hensen, H. Bernien, S. B. van Dam, M. S. Blok, T. H. Taminiau, M. J. Tiggelman, R. N. Schouten, M. Markham, D. J. Twitchen, and R. Hanson, Science 345, 532 (2014).

[43] N. Somaschi, V. Giesz, L. De Santis, J. C. Loredo, M. P. Almeida, G. Hornecker, S. L. Portalupi, T. Grange, C. Antón, J. Demory, C. Gómez, I. Sagnes, N. D. Lanzillotti-Kimura, A. Lemaítre, A. Auffeves, A. G. White, L. Lanco, and P. Senellart, Nat. Photonics 10, 340 (2016).

[44] H. Wang, Z.-C. Duan, Y.-H. Li, S. Chen, J.-P. Li, Y.-M. He, M.-C. Chen, Y. He, X. Ding, C.-Z. Peng, C. Schneider, M. Kamp, S. Höfling, C.-Y. Lu, and J.-W. Pan, Phys. Rev. Lett. 116, 213601 (2016).

[45] J. H. Prechtel, A. V. Kuhlmann, J. Houel, A. Ludwig, S. R. Valentin, A. D. Wieck, and R. J. Warburton, Nat. Mater. 15, 981 (2016).

[46] C. Monroe, R. Raussendorf, A. Ruthven, K. R. Brown, P. Maunz, L.-M. Duan, and J. Kim, Phys. Rev. A 89, 022317 (2014).

[47] D. Kim, S. G. Carter, A. Greilich, A. Bracker, and D. Gammon, Nat. Phys. 7, 223 (2011).

[48] D. Kim, A. A. Kiselev, R. S. Ross, M. T. Rakher, C. Jones, and T. D. Ladd, Phys. Rev. Applied 5, 024014 (2016). 